\title{
Summary of April 10, 2017 Lattice
}

\author{
J.S. Berg
}

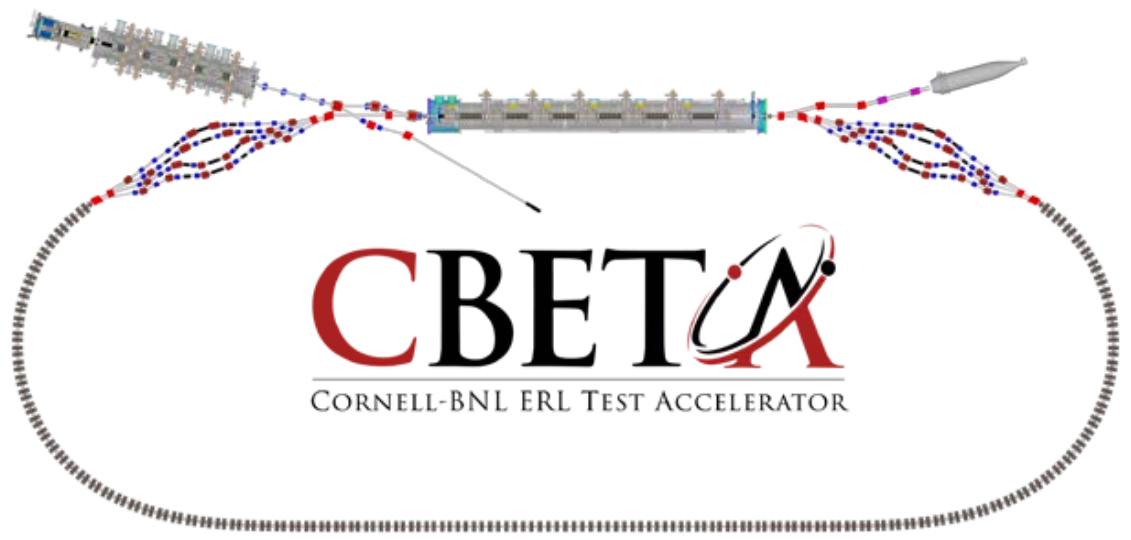

Collider-Accelerator Department, Brookhaven National Laboratory, Upton NY 11973 U.S. Department of Energy

Office of Science, Office of Nuclear Physics

Cornell Laboratory for Accelerator-Based Sciences and Education, Ithaca, NY 14850

Funded by NYSERDA contract 102192

Notice: This document has been authorized by employees of Brookhaven Science Associates, LLC under Contract No. DE-SC0012704 with the U.S. Department of Energy. The United States Government retains a non-exclusive, paid-up, irrevocable, world-wide license to publish or reproduce the published form of this document, or allow others to do so, for United States Government purposes. 


\section{DISCLAIMER}

This report was prepared as an account of work sponsored by an agency of the United States Government. Neither the United States Government nor any agency thereof, nor any of their employees, nor any of their contractors, subcontractors, or their employees, makes any warranty, express or implied, or assumes any legal liability or responsibility for the accuracy, completeness, or any third party's use or the results of such use of any information, apparatus, product, or process disclosed, or represents that its use would not infringe privately owned rights. Reference herein to any specific commercial product, process, or service by trade name, trademark, manufacturer, or otherwise, does not necessarily constitute or imply its endorsement, recommendation, or favoring by the United States Government or any agency thereof or its contractors or subcontractors. The views and opinions of authors expressed herein do not necessarily state or reflect those of the United States Government or any agency thereof. 


\title{
Summary of April 10, 2017 Lattice
}

\author{
J. Scott Berg ${ }^{1}$ \\ Brookhaven National Laboratory; P. O. Box 5000; Upton, NY, 11973-5000; USA
}

\begin{abstract}
We present a summary of the April 17, 2017 CBETA Lattice.

Table 1: Machine parameters

\begin{tabular}{lr}
\hline Total energy, injection $(\mathrm{MeV})$ & 6 \\
Total energy, pass 1 (MeV) & 42 \\
Total energy, pass 2 (MeV) & 78 \\
Total energy, pass 3 (MeV) & 114 \\
Total energy, pass $4(\mathrm{MeV})$ & 150 \\
RF frequency (GHz) & $1.29994000 \mathrm{GHz}$ \\
Harmonic number, normal pass & 343 \\
\hline
\end{tabular}
\end{abstract}

\section{Introduction}

Table 1 gives important high-level parameters for the machine. A drawing of the CBETA lattice is shown in Fig. 1. The machine consists of a number of sections:

Injector (IN) Production of the beam, acceleration to $6 \mathrm{MeV}$, and transport to the beginning of the linac. This is already in place and operating, and little more detail will be provided here.

Linac (LA) A $1.3 \mathrm{GHz}$ superconducting linac, accelerating electrons nominally by $36 \mathrm{MeV}$. This is already in place and operating, and little more detail will be provided here, other than the fact that the precise operating frequency will be $1.29994000 \mathrm{GHz}$.

Beam Stop (BS) A transport line for the final $6 \mathrm{MeV}$ decelerated beam.

Spreaders (SX/RX) SX will capture the beams from the linac at the four nominal design energies, match the betatron functions, and steer the orbits so that after the beams are recombined, they arrive on the periodic orbits in the FFAG arc. RX reverses this process, taking the beams from the FFAG arc back to the linac.

\footnotetext{
Email address: jsberg@bnl.gov (J. Scott Berg)

${ }^{1}$ This manuscript has been authored by employees of Brookhaven Science Associates, LLC under Contract No. DE-SC0012704 with the U.S. Department of Energy. The United States Government retains a non-exclusive, paid-up, irrevocable, world-wide license to publish or reproduce the published form of this manuscript, or allow others to do so, for United States Government purposes.
}

Arc (FA/FB) The FFAG arc returning the beams coming out of and going back into the the spreaders.

Straight (ZA/ZB) A straight beamline similar to the FFAG arc, returning the beam across the distance parallel to the linac.

Transition (TA/TB) An adiabatic transition between the FFAG arc and the straight, bringing the full energy range of beams from the arcs onto the axis of the straight.

The arc, straight, and transition sections are detailed in a separate document, with the exception that I will detail some modifications of individual magnets near the spreader.

\section{Spreaders}

There are four SX lines and four corresponding RX lines, corresponding to the four design energies. They are numbered 1 through 4, forom lowest to highest energies, and designated S1, S2, etc. A detailed view of the SX lines is shown in Fig. 2. The first three lines have two different configurations, depending on whether the energy recovery linac is run with that pass at its maximum energy or not. Each spreader line has one configuration when it is used for the highest energy (recovery mode), and a different configuration when it is lower than the highest energy (non-recovery mode). This reconfiguration requires moving the magnets and beam pipe. The current lattice files have configurations for 1 pass and 4 pass energy recovery. Configurations for 2 and 3 pass recovery still need to be designed.

Just before the SX lines, there is a dipole (LA.DEMER. BEN01) to bend the $6 \mathrm{MeV}$ beam to the beam stop line that all beams will pass through. When running in the fractional arc test (FAT) configuration, this magnet will not be present. A study of the lattice in this configuration has yet to be done.

Each spreader line has 8 quadrupoles for betatron and dispersion matching plus $R_{56}$ control. All quadrupoles are $20 \mathrm{~cm}$ long. 


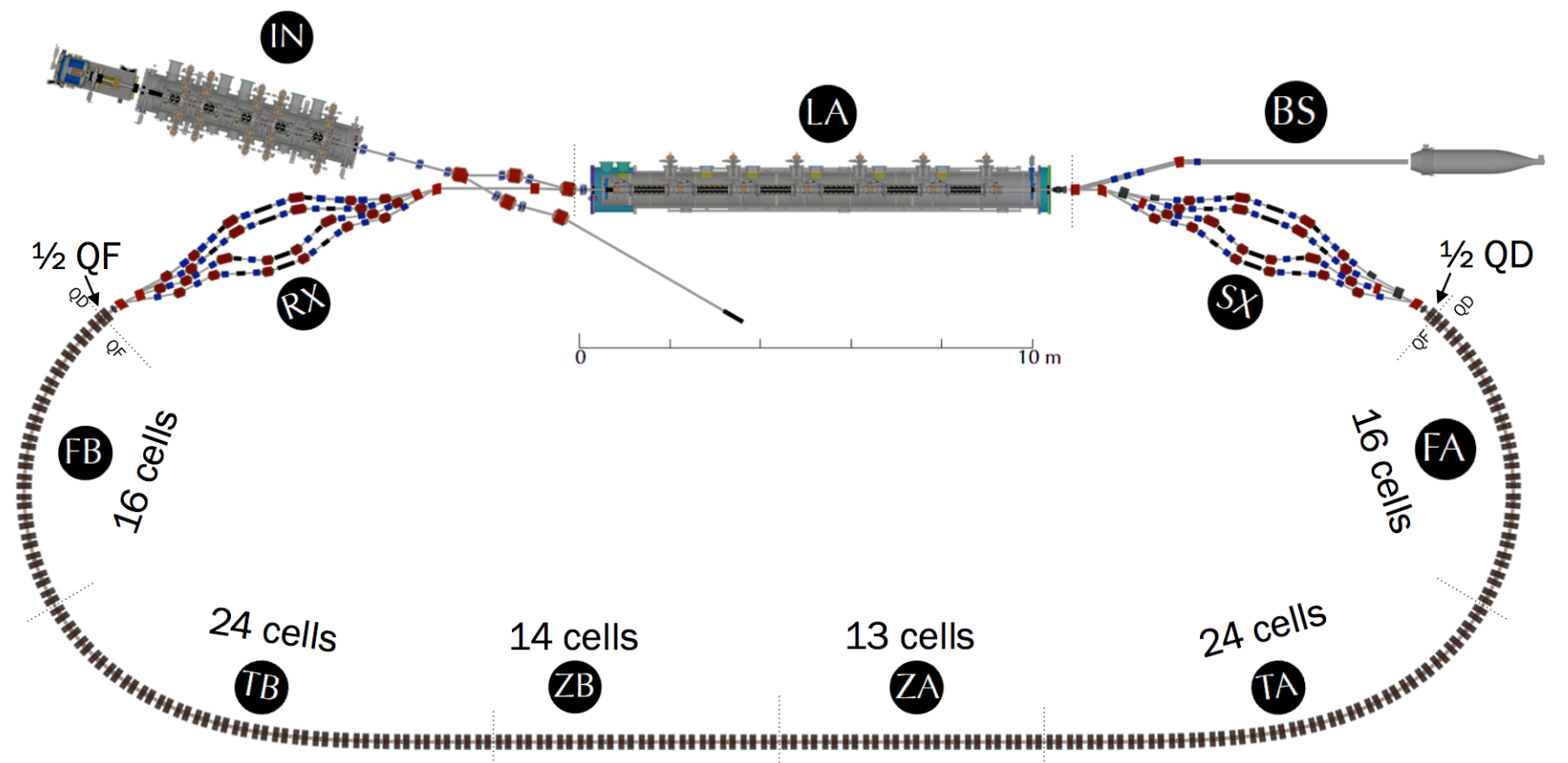

Figure 1: Overview of the CBETA lattice.

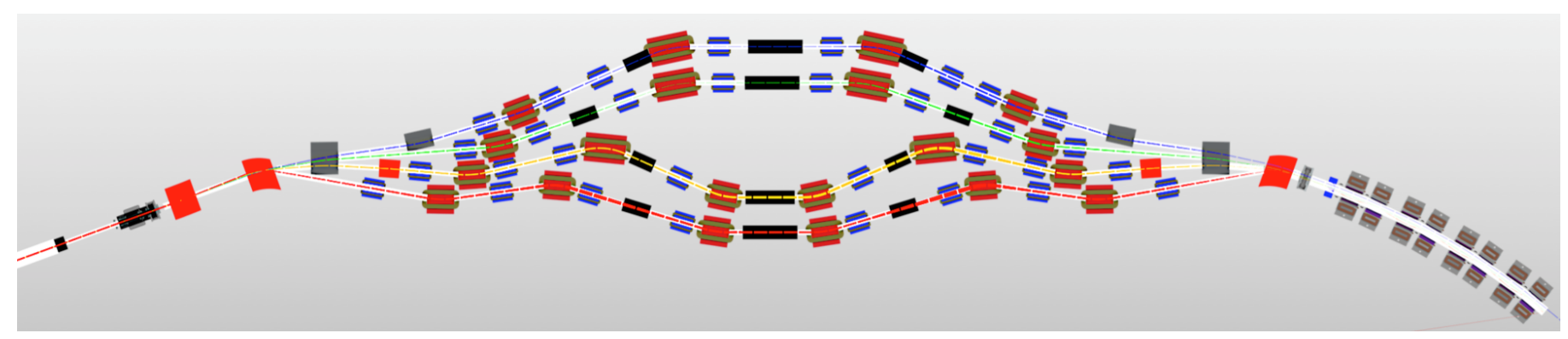

Figure 2: Detail view of the SX spreader line. 
Table 2: Common dipole parameters.

\begin{tabular}{lrrr}
\hline & $\begin{array}{r}\text { Energies } \\
(\mathrm{MeV})\end{array}$ & $\begin{array}{r}\text { Length } \\
(\mathrm{m})\end{array}$ & $\begin{array}{r}\text { Field } \\
(\mathrm{T})\end{array}$ \\
\hline IN.MERGE.BEN03 & $6-150$ & 0.32 & +0.0160 \\
LA.DEMER.BEN01 & $6-150$ & 0.20 & +0.0258 \\
S1.BEN01 & $42-150$ & 0.20 & -0.3760 \\
S1.BEN08 & $42-150$ & 0.20 & -0.3502 \\
R1.BEN01 & $42-150$ & 0.20 & -0.3502 \\
R1.BEN08 & $42-150$ & 0.20 & -0.3502 \\
R1.BEN99 & $42-150$ & 0.20 & -0.0258 \\
S2.BEN01 & $78-150$ & 0.20 & -0.1676 \\
S2.BEN10 & $78-150$ & 0.20 & -0.1676 \\
R2.BEN01 & $78-150$ & 0.20 & -0.1676 \\
R2.BEN10 & $78-150$ & 0.20 & -0.1676 \\
\hline
\end{tabular}

Numbering of elements in spreaders starts with smaller numbers at the linac end unless otherwise indicated.

From the design report, we intend to have four vertical correcters per spreader line. They are not currently in the lattice.

The beam pipes between the central four dipoles of each line are connected by sliding joints to allow for small path length adjustments.

\subsection{Common Dipoles}

Several dipoles are shared by multiple beams. IN. MERGE.BEN03 merges the injected beam at the linac, while LA.DEMER.BEN01 serves to send the low energy beam to the dump after the linac. IN.MERGE.BEN03 is currently in place on the floor. LA.DEMER.BEN01 will not be in place for the fractional arc test. S1.BEN01 separates the beams at the entrance to the spreader into multipole lines, and S1.BEN08 recombines them before entering the FFAG section. Since the diagnostic beam line for the injector requires a greater space between R1.BEN01 and IN.MERGE.BEN03 than there is between LA.DEMER. BEN01 and S1.BEN01, an extra dipole, R1.BEN99, is inserted between R1.BEN01 and IN.MERGE.BEN03 assist with steering while allowing the $\mathrm{Rn}$ lines to be nearly symmetric to the Sn lines. S2.BEN01, S2.BEN10, R2.BEN01, and R2.BEN10 are septa that bend the three highest energies. Table 2 gives the fields and lengths of these dipoles. The common dipoles nominally create a 30 degree bend angle for the $42 \mathrm{MeV}$ beam at each end of the spreaders. Similarly, the common dipoles nominally create a 23 degree bend angle for the $78 \mathrm{MeV}$ beam at each end of the spreaders.

\subsection{Individual Lines}

S1 and R1 each have 6 dipoles that are not shared with other lines. When doing energy recovery for more than one pass (non-recovery mode), these dipoles are set to bend by specific angles, and no additional tuning is applied to them in the design. Table 3 gives the dipole parameters in non-recovery mode for these lines, and Table 4 gives the
Table 3: S1/R1 non-common dipole parameters, non-recovery.

\begin{tabular}{lrrr}
\hline & Length $(\mathrm{m})$ & Angle (deg.) & Field $(\mathrm{T})$ \\
\hline S1.BEN02 & 0.20 & -18 & +0.2200 \\
S1.BEN03 & 0.20 & +25 & -0.3056 \\
S1.BEN04 & 0.20 & -17 & +0.2078 \\
S1.BEN05 & 0.20 & -17 & +0.2078 \\
S1.BEN06 & 0.20 & +25 & -0.3056 \\
S1.BEN07 & 0.20 & -18 & +0.2200 \\
R1.BEN02 & 0.20 & -18 & +0.2200 \\
R1.BEN03 & 0.20 & +25 & -0.3056 \\
R1.BEN04 & 0.20 & -17 & +0.2078 \\
R1.BEN05 & 0.20 & -17 & +0.2078 \\
R1.BEN06 & 0.20 & +25 & -0.3056 \\
R1.BEN07 & 0.20 & -18 & +0.2200 \\
\hline
\end{tabular}

Table 4: S1/R1 quadrupole gradients, non-recovery.

\begin{tabular}{lrrr}
\hline & $\begin{array}{r}\text { Gradient } \\
(\mathrm{T} / \mathrm{m})\end{array}$ & & $\begin{array}{r}\text { Gradient } \\
(\mathrm{T} / \mathrm{m})\end{array}$ \\
\hline S1.QUA01 & -0.2953 & R1.QUA01 & -0.4413 \\
S1.QUA02 & +0.8173 & R1.QUA02 & +0.9319 \\
S1.QUA03 & -1.1328 & R1.QUA03 & -0.9613 \\
S1.QUA04 & +0.6881 & R1.QUA04 & +0.3480 \\
S1.QUA05 & +2.0911 & R1.QUA05 & +1.8964 \\
S1.QUA06 & -1.4607 & R1.QUA06 & -1.3669 \\
S1.QUA07 & +1.2341 & R1.QUA07 & +0.8416 \\
S1.QUA08 & -0.5383 & R1.QUA08 & -0.0955 \\
\hline
\end{tabular}

Table 5: S1/R1 dipoles changed for recovery mode. BEN61 replaces BEN03, BEN62 replaces BEN04, etc. BEN02 and BEN07 are unchanged.

\begin{tabular}{lrrr}
\hline & Length $(\mathrm{m})$ & Angle (deg.) & Field (T) \\
\hline R1.BEN61 & 0.20 & +19.03 & -0.2326 \\
R1.BEN62 & 0.20 & -11.03 & +0.1348 \\
R1.BEN63 & 0.20 & -11.03 & +0.1348 \\
R1.BEN64 & 0.20 & +19.03 & -0.2326 \\
S1.BEN61 & 0.20 & +19.03 & -0.2326 \\
S1.BEN62 & 0.20 & -11.03 & +0.1348 \\
S1.BEN63 & 0.20 & -11.03 & +0.1348 \\
S1.BEN64 & 0.20 & +19.03 & -0.2326 \\
\hline
\end{tabular}

Table 6: S1/R1 quadrupole gradients, recovery.

\begin{tabular}{lrrr}
\hline & $\begin{array}{r}\text { Gradient } \\
(\mathrm{T} / \mathrm{m})\end{array}$ & & $\begin{array}{r}\text { Gradient } \\
(\mathrm{T} / \mathrm{m})\end{array}$ \\
\hline S1.QUA01 & -0.6245 & R1.QUA01 & -0.5864 \\
S1.QUA02 & +1.1663 & R1.QUA02 & +1.2760 \\
S1.QUA03 & -0.6935 & R1.QUA03 & -0.7145 \\
S1.QUA04 & +0.4160 & R1.QUA04 & +0.5310 \\
S1.QUA05 & +2.0362 & R1.QUA05 & +1.8314 \\
S1.QUA06 & -1.1840 & R1.QUA06 & -1.1044 \\
S1.QUA07 & +1.3461 & R1.QUA07 & +0.9622 \\
S1.QUA08 & -0.4832 & R1.QUA08 & +0.1230 \\
\hline
\end{tabular}


Table 7: S2/R2 non-common dipole parameters, non-recovery.

\begin{tabular}{lrrr}
\hline & Length $(\mathrm{m})$ & Angle (deg.) & Field $(\mathrm{T})$ \\
\hline S2.BEN02 & 0.15 & +2.00 & -0.0605 \\
S2.BEN03 & 0.20 & -18.00 & +0.4087 \\
S2.BEN04 & 0.30 & +37.00 & -0.5600 \\
S2.BEN05 & 0.20 & -24.00 & +0.5449 \\
S2.BEN06 & 0.20 & -24.00 & +0.5449 \\
S2.BEN07 & 0.30 & +36.77 & -0.5565 \\
S2.BEN08 & 0.20 & -17.63 & +0.4002 \\
S2.BEN09 & 0.15 & +2.00 & -0.0605 \\
R2.BEN02 & 0.15 & +2.00 & -0.0605 \\
R2.BEN03 & 0.20 & -18.00 & +0.4086 \\
R2.BEN04 & 0.30 & +37.00 & -0.5600 \\
R2.BEN05 & 0.20 & -24.00 & +0.5449 \\
R2.BEN06 & 0.20 & -24.00 & +0.5449 \\
R2.BEN07 & 0.30 & +35.53 & -0.5378 \\
R2.BEN08 & 0.20 & -15.90 & +0.3610 \\
R2.BEN09 & 0.15 & +2.00 & -0.0605 \\
\hline
\end{tabular}

Table 8: S2/R2 quadrupole gradients, non-recovery.

\begin{tabular}{lrrr}
\hline & $\begin{array}{r}\text { Gradient } \\
(\mathrm{T} / \mathrm{m})\end{array}$ & & $\begin{array}{r}\text { Gradient } \\
(\mathrm{T} / \mathrm{m})\end{array}$ \\
\hline S2.QUA01 & -0.5638 & R2.QUA01 & -0.5665 \\
S2.QUA02 & +0.6729 & R2.QUA02 & +0.6433 \\
S2.QUA03 & -1.4963 & R2.QUA03 & -1.2778 \\
S2.QUA04 & +0.8750 & R2.QUA04 & +0.5591 \\
S2.QUA05 & +0.3653 & R2.QUA05 & +0.7014 \\
S2.QUA06 & -2.1499 & R2.QUA06 & -2.3046 \\
S2.QUA07 & +1.6422 & R2.QUA07 & +1.6184 \\
S2.QUA08 & +0.1734 & R2.QUA08 & +0.6334 \\
\hline
\end{tabular}

quadrupole gradients. Tables 5 and 6 give the corresponding parameters in recovery mode. Note that only some dipoles change in recovery mode.

$\mathrm{S} 2$ and $\mathrm{R} 2$ each have 8 dipoles that are not shared with other lines. The dipoles are set to specific angles, then BEN07 and BEN08 are adjusted make slight steering corrections. Their dipole parameters are given in Table 7 , and their quadrupole gradients are given in Table 8 .

S3 and R3 each have 4 dipoles that are not shared with other lines. BEN02 and BEN03 have defined angles, the remaining dipoles are adjusted to give the desired total

Table 9: S3/R3 non-common dipole parameters, non-recovery.

\begin{tabular}{lrrr}
\hline & Length $(\mathrm{m})$ & Angle (deg.) & Field (T) \\
\hline S3.BEN01 & 0.20 & -15.66 & +0.5196 \\
S3.BEN02 & 0.30 & +20.00 & -0.4425 \\
S3.BEN03 & 0.30 & +20.00 & -0.4425 \\
S3.BEN04 & 0.20 & -15.66 & +0.5195 \\
R3.BEN01 & 0.20 & -15.73 & +0.5218 \\
R3.BEN02 & 0.30 & +20.00 & -0.4425 \\
R3.BEN03 & 0.30 & +20.00 & -0.4425 \\
R3.BEN04 & 0.20 & -15.50 & +0.5142 \\
\hline
\end{tabular}

Table 10: S3/R3 quadrupole gradients.

\begin{tabular}{lcrr}
\multicolumn{3}{c}{$\begin{array}{c}\text { Gradient } \\
(\mathrm{T} / \mathrm{m})\end{array}$} & $\begin{array}{r}\text { Gradient } \\
(\mathrm{T} / \mathrm{m})\end{array}$ \\
\hline S3.QUA01 & -1.4116 & R3.QUA01 & -1.5254 \\
S3.QUA02 & +0.2650 & R3.QUA02 & +0.5056 \\
S3.QUA03 & +0.4364 & R3.QUA03 & +0.6096 \\
S3.QUA04 & -1.6250 & R3.QUA04 & -1.8527 \\
S3.QUA05 & +1.1217 & R3.QUA05 & +0.2872 \\
S3.QUA06 & -0.7900 & R3.QUA06 & -0.0612 \\
S3.QUA07 & -1.6307 & R3.QUA07 & -2.1204 \\
S3.QUA08 & +0.7756 & R3.QUA08 & +1.2333 \\
\hline
\end{tabular}

Table 11: S4/R4 non-common dipole parameters.

\begin{tabular}{lrrr}
\hline & Length $(\mathrm{m})$ & Angle (deg.) & Field (T) \\
\hline S4.BEN01 & 0.20 & -9.00 & +0.3930 \\
S4.BEN02 & 0.20 & -6.89 & +0.3011 \\
S4.BEN03 & 0.30 & +24.00 & -0.6986 \\
S4.BEN04 & 0.30 & +24.00 & -0.6986 \\
S4.BEN05 & 0.20 & -6.85 & +0.2992 \\
S4.BEN06 & 0.20 & -9.00 & +0.3930 \\
R4.BEN01 & 0.20 & -9.00 & +0.3930 \\
R4.BEN02 & 0.20 & -6.97 & +0.3045 \\
R4.BEN03 & 0.30 & +24.00 & -0.6986 \\
R4.BEN04 & 0.30 & +24.00 & -0.6986 \\
R4.BEN05 & 0.20 & -7.13 & +0.3113 \\
R4.BEN06 & 0.20 & -9.00 & +0.3930 \\
\hline
\end{tabular}

bend angle including common magnets, and also to provide some steering of the beam. The dipole parameters are given in Table 9, and their quadrupole gradients are given in Table 10.

S4 and R4 each have 6 dipoles that are not shared with other lines. BEN01 and BEN06 are septa. BEN01, BEN03, BEN04, and BEN06 have defined angles, while the remaining dipoles are adjusted to give the desired total bend angle including common magnets, and also to provide some steering of the beam. The dipole parameters are given in Table 11, and their quadrupole gradients are given in Table 12.

Table 12: S4/R4 quadrupole gradients.

\begin{tabular}{lcrr}
\hline & $\begin{array}{c}\text { Gradient } \\
(\mathrm{T} / \mathrm{m})\end{array}$ & & $\begin{array}{r}\text { Gradient } \\
(\mathrm{T} / \mathrm{m})\end{array}$ \\
\hline S4.QUA01 & +0.3990 & R4.QUA01 & +0.0072 \\
S4.QUA02 & -2.7819 & R4.QUA02 & -2.5713 \\
S4.QUA03 & +2.6538 & R4.QUA03 & +2.9938 \\
S4.QUA04 & -3.4843 & R4.QUA04 & -3.9228 \\
S4.QUA05 & +1.4835 & R4.QUA05 & +0.0902 \\
S4.QUA06 & -3.3893 & R4.QUA06 & -3.8358 \\
S4.QUA07 & -0.3454 & R4.QUA07 & +0.4393 \\
S4.QUA08 & +2.3217 & R4.QUA08 & +2.0596 \\
\hline
\end{tabular}




\begin{tabular}{lr}
\hline & $\begin{array}{r}\text { Gradient } \\
(\mathrm{T} / \mathrm{m})\end{array}$ \\
\hline DU.QUA01 & -0.6652 \\
DU.QUA02 & +0.6297 \\
DU.QUA03 & -0.6749 \\
DU.QUA04 & -0.1340 \\
\hline
\end{tabular}

\section{FFAG Arcs (FA/FB)}

At the beginning of FA, there is one special half-length $\mathrm{BD}$, centered on the beam pipe. It is $12 \mathrm{~mm}$ from a standard $42 \mathrm{~mm}$ BPM block. $16 \mathrm{~mm}$ downstream from that BPM block, there is the first QF in a standard FFAG cell, offset an additional $3 \mathrm{~mm}$ to the outside. The longitudinal spacing then continues as usual in the arc. The first standard BD (FA.QUA02) is offset inward by $3.2 \mathrm{~mm}$, the second BD (FA.QUA04) is offset outward by $0.5 \mathrm{~mm}$, and the remaining quadrupoles are in their standard positions.

Similarly, at the end of FB, there is a half-length QF, $12 \mathrm{~mm}$ downstream from a standard BPM block, then $16 \mathrm{~mm}$ to a standard BD (FB.QUA32) offset inward by $4.3 \mathrm{~mm}$, then a standard longitudinal geometry upstream from there. There are additional offsets upstream in the next QF (2.4 mm outward). The lattice file has smaller offsets in the for most of the remaining upstream BDs in FB.

\section{Beam Stop (BS)}

The beam stop line has four $0.15 \mathrm{~m}$ quadrupoles and one $0.2 \mathrm{~m}$ dipole. The dipole bends by 15 degrees and has an field of $0.0261 \mathrm{~T}$. Quadrupole gradients are given in Table 13.

\section{Performance}

Figure 3 shows the emittance through four passes of energy recovery. Figure 4 shows the normalized emittance through four passes of energy recovery. Emittance growth is negligible.

\section{Appendix A. Items Remaining}

- Recovery mode design for S2/R2

- Recovery mode design for S3/R3

- Move S2.QUA02/QUA07 (and similarly for R2) to resolve physical conflict with S3.BEN01/BEN04

- Add vertical correctors to spreaders 

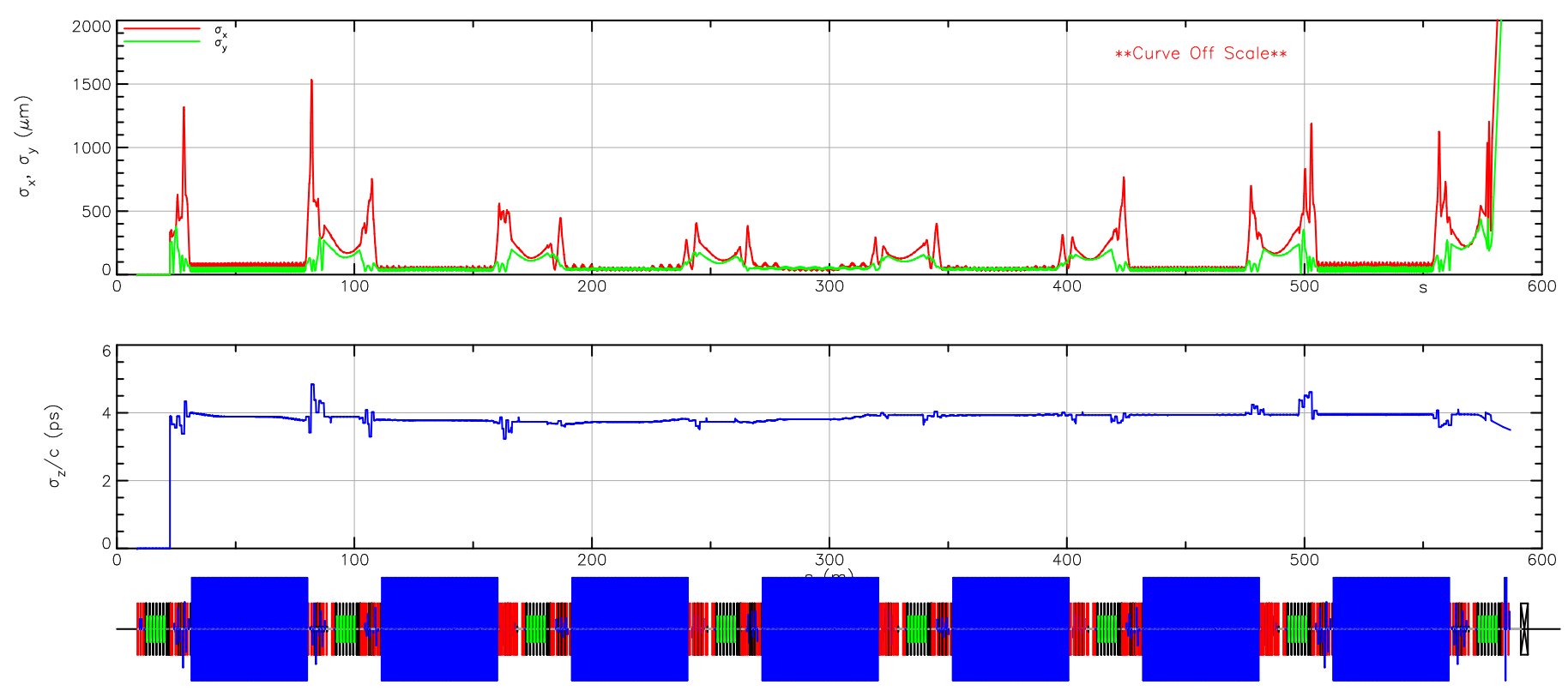

Figure 3: Beam size and bunch length through 4-pass energy recovery.
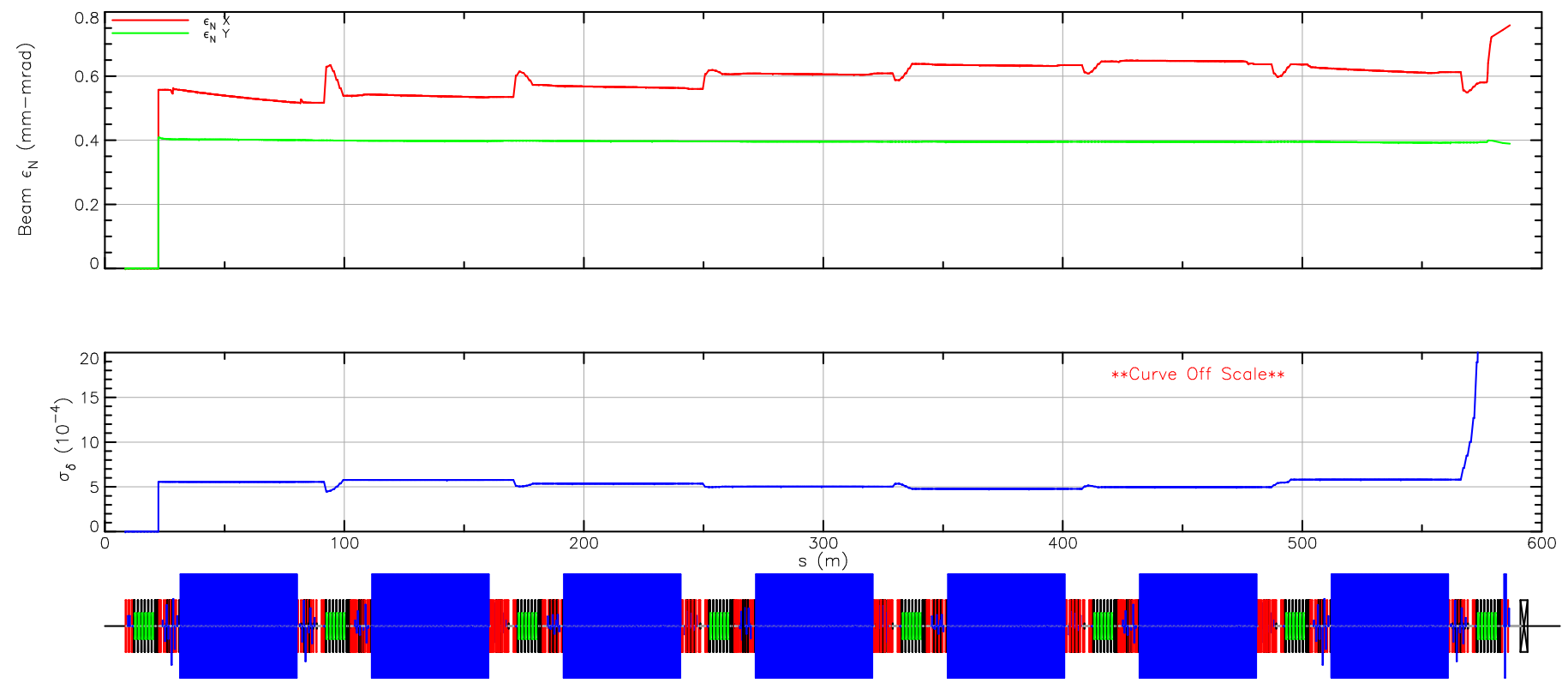

Figure 4: Normalized beam emittance and energy spread through 4-pass energy recovery. 